\title{
How common is tooth pain after root canal treatment?
}

\author{
Abstracted from \\ Nixdorf DR, Moana-Filho EJ, Law AS, McGuire LA, Hodges JS, John MT. \\ Frequency of persistent tooth pain after root canal therapy: a systematic review and meta-analysis. \\ J Endod 2010; 36: 224-230 \\ Address for correspondence: Dr Donald Nixdorf, University of Minnesota, 6-320 Moos Tower \\ 515 Delaware Street SE, Minneapolis, MN 55455, USA. E-mail address: nixdorf@umn.edu
}

\section{Questions: What is the frequency of tooth pain at 6 months in patients undergoing root canal therapy?}

Data sources Medline, the Cochrane Library, TRIP database and Google Scholar, hand searching of articles, reviews and textbooks. Study selection Articles in any language about endodontic procedures, (initial treatment or retreatment and surgical or nonsurgical but not pulpotomy, partial pulpectomy or pulp capping) in permanent teeth that reported on pain at a minimum of six months were included. The main outcome was the presence of all-cause pain, with no differentiation on the basis of aetiologies. The outcome of all-cause pain was considered positive if reported by either the patient or the practitioner. Pain could be spontaneous or provoked by biting, palpation or percussion.

Data extraction and synthesis Data were extracted independently by two reviewers and STROBE criteria (www.strobe-statement.org) used to assess quality. A summary estimate of persistent all-cause pain frequency was established by using a random-effects meta-analysis. The influence of treatment approach (surgical/nonsurgical), longitudinal study design (prospective/retrospective), follow-up rate and duration, initial treatment versus retreatment, and quality of reporting on the pain frequency estimate was assessed in subgroup analyses.

Results 26 studies that included 5777 teeth with 2996 (51.9\%) having follow-up pain information met the inclusion criteria. 168 teeth with pain were identified giving a frequency of $5.3 \%$ (95\% confidence interval, $3.5 \%-7.2 \%, p<0.001)$ for persistent all-cause pain. High and statistically significant heterogeneity among studies $\left(1^{2}=80 \%\right)$ was present. In subgroup analysis, prospective studies had a higher pain frequency (7.6\%) than retrospectives studies $(0.9 \%)$. Quality of study reporting was identified as the most influential reason for study heterogeneity.

Conclusions The frequency of all-cause persistent pain after endodontic procedures was estimated to be $5.3 \%$, with higher report quality studies suggesting $>7 \%$.

\section{Commentary}

When reviewing the studies on endodontic treatment, it quickly becomes apparent that outcome definitions and classification have been inconsistent. This has resulted in considerable variability of the reported success rate of different treatment modalities. ${ }^{1}$

However, there is considerable interest in characterising the outcome of various endodontic modalities to compare with other treatment options and to assess the clinical performance of a given treatment strategy. This study is of particular importance because it estimated the frequency of persistent pain, regardless of aetiology, after endodontic treatment. It is a very well designed systematic review and the authors calculated elegantly the estimate of pain frequency, taking into consideration the overall heterogeneity: on the other hand, they identified potential design issues and limitations.

Though the search for an objective method to characterise endodontic treatment outcome is ongoing, radiographic comparison remains the current gold standard, largely because of its objectivity and correlation with the microbiological, immunological and neurological healing kinetics. In considering this review the reader needs to bear in mind the great variation in treatment procedures. In the present study treatment ranged over 34 years (between 1975 and 2009), during which dramatic changes in the philosophy and technology of endodontic treatment took place. Add to this a range of other variables such as methods of instrumentation, obturation, asepsis, and many others. ${ }^{3}$ Also there is the possibility of a new infection after the primary treatment leading to another wave of symptoms, particularly after this length of time. ${ }^{4}$ So while the estimate of pain frequency presented in a review for the first time interesting, it might not constitute the current position of today's persistent tooth pain after endodontic therapy.

A question not addressed by the review is whether there is any correlation between apical rarefaction and pain. This and the role of biological mechanisms for persistent apical periodontitis needs to be researched in order to characterise the course of endodontic diseases to further improve upon the efficiency of assessing the treatment outcome.

\section{Practice point}

-While contemporary endodontic standards recognise absence of symptoms as crucial to successful treatment outcome; clarity on the use of the terms healing, diseased, function and retention is needed to improve outcome prediction.

\section{Khaled Balto}

Faculty of Dentistry, King Abdulaziz University, Jeddah, Saudi Arabia.

1. Friedman S. Treatment outcomes and prognosis of endodontic therapy. In Orstavik D, Pitt Ford TR (eds) Essential Endodontology: prevention and treatment of apical periodontitis. pp 367-391. Oxford: Blackwell Science 1998.

2. Friedman $\mathrm{S}$. The prognosis and expected outcome of apical surgery. Endod Topics 2006; 11: 219-262.

3. Fristad I, Molven $\mathrm{O}$, Halse A. Nonsurgically retreated root-filled teeth-radiographic findings after 20-27 years. Int Endod J 2004; 37: 12-18.

4. Haapasalo $M$, Udnaes T, Endal U. Persistent, recurrent, and acquired infection of the root canal system post-treatment. Endod Topics 2003; 6: 29-56.

Evidence-Based Dentistry (2010) 11, 114. doi:10.1038/sj.ebd.6400758 